\title{
Single Arm Phase I/II Study of Everolimus and Intravesical Gemcitabine in Patients with Primary or Secondary Carcinoma In Situ of the Bladder who failed Bacillus Calmette Guerin (NCT01259063)
}

Guido Dalbagni $^{\mathrm{a}, *}$, Nicole Benfante ${ }^{\mathrm{b}}$, Daniel D. Sjoberg ${ }^{\mathrm{b}}$, Bernard H. Bochner ${ }^{\mathrm{a}}$, S. Machele Donat $^{\mathrm{a}}$, Harry W. Herr ${ }^{\mathrm{a}}$, Asia S. Mc Coy ${ }^{\mathrm{c}}$, Alicia J. Fahrner ${ }^{\mathrm{a}}$, Caitlyn Retinger ${ }^{\mathrm{a}}$, Jonathan E. Rosenberg ${ }^{\mathrm{c}}$ and Dean F. Bajorin ${ }^{\mathrm{c}}$

${ }^{a}$ Urology Service, Department of Surgery, Memorial Sloan Kettering Cancer Center, New York, NY, USA

${ }^{\mathrm{b}}$ Department of Epidemiology and Biostatistics, Memorial Sloan Kettering Cancer Center, New York, NY, USA

${ }^{\mathrm{c}}$ Genitourinary Oncology Service, Department of Medicine, Memorial Sloan Kettering Cancer Center, New York, $N Y, U S A$

\begin{abstract}
.
Background: Standard treatment for BCG-refractory urothelial cancer is radical cystectomy. Identification of active agents is clearly warranted.

Objective: To determine a safe dose of oral everolimus in combination with standard intravesical gemcitabine and to evaluate the efficacy of this combination.

Methods: Patients with carcinoma in situ refractory to intravesical bacillus Calmette-Guérin and refusing cystectomy were eligible. Patients in the phase I part of the trial received one of three dose levels of oral everolimus. Patients also received a fixed dose of intravesical gemcitabine. Maintenance everolimus was given for 12 months in patients achieving a complete response confirmed by cystoscopy and cytology. Patients in phase II received continuous everolimus administered at $10 \mathrm{mg}$ daily with intravesical gemcitabine followed by everolimus maintenance for 12 months of total therapy. The enrollment goal for the phase II was 33 patients.

Results: 14 patients were enrolled in phase I of the trial. 23 patients were enrolled in phase II of the trial and 19 were evaluable for primary and secondary endpoints. Four patients withdrew consent prior to treatment initiation. Of the 19 patients evaluable for response, $3(16 \%, 95 \%$ confidence interval [CI] 3\%-40\%) were disease free at $1 \mathrm{yr}$. The probability of RFS was $20 \%$ $(95 \%$ CI $5 \%-42 \%)$ at 12 months. Ten patients out of 19 had grade 3 or greater toxicity events. Seven withdrew consent or were taken off study.

Conclusions: Many patients withdrew, and enrollment was halted. Continuous oral everolimus plus intravesical gemcitabine was not well tolerated in this patient population where the threshold for tolerability is low.
\end{abstract}

Keywords: Gemcitabine, urothelial cancer, bladder cancer, intravesical chemotherapy, phase II, everolimus, mTOR inhibitor, RAD001

\footnotetext{
*Correspondence to: Guido Dalbagni, MD, Urology Service, Department of Surgery, Memorial Sloan Kettering Cancer Center, Sidney Kimmel Center, 353 East 68th Street, New York, NY
} 


\section{INTRODUCTION}

Bladder cancer is the fifth most common cancer in the United States [1]. Seventy percent of bladder tumors are non-muscle-invasive (formerly called "superficial") at presentation and include carcinoma in situ (cis), Ta and T1 disease. Despite conservative management by endoscopic resection, $60 \%$ to $70 \%$ of superficial tumors recur [2], and $20 \%$ to $30 \%$ of recurrent tumors will progress to a higher stage or grade [3]. Bacillus Calmette-Guérin (BCG) is the most effective agent in the treatment of cis [4] and decreases the rate of progression [5-10]. However, only two-thirds of patients respond to BCG, and onethird of the responders will develop recurrent disease, which is associated with a poor prognosis $[11,12]$. Given that the only other accepted standard treatment for BCG-refractory urothelial cancer is radical cystectomy, identification of active agents in this disease is clearly warranted.

Gemcitabine (2',2'-difluoro-2'-deoxycytidine; Gemzar, Eli Lilly and Co, Indianapolis, IN) is a deoxycytidine analog with a broad spectrum of antitumor activity. This agent is highly effective (overall response rates ranging from $22.5 \%$ to $28 \%$ ) and well tolerated as both first- and second-line, single-agent therapy for the treatment of metastatic urothelial carcinoma [13-15].

We reported a phase II study of intravesical gemcitabine given twice a wk for $3 \mathrm{wk}$, followed by a second 3-wk cycle after a wk of rest, in a heavily pretreated population with BCG-refractory urothelial carcinoma. This study demonstrated that intravesical gemcitabine was well tolerated and had modest efficacy. Of the 30 patients, $15(50 \%, 95 \%$ confidence interval [CI] 32\%-68\%) achieved an initial complete response (CR). Twelve patients recurred with a median recurrence-free survival (RFS) of $3.6 \mathrm{mo}$ (95\% CI 2.9 mo-11.0 mo). Two patients remained in CR at 23 months and 29 months. The 1-yr RFS for patients with a CR was $21 \%(95 \%$ CI $0 \%-43 \%)$ and $10 \%$ for the whole cohort [16].

Everolimus is a novel oral derivative of rapamycin which has shown activity in multiple malignancies including modest activity in bladder cancer $[17,18]$. This mTOR inhibitor has been shown to inhibit protein synthesis and growth of bladder cancer cells in vitro [19]. mTOR inhibitors have also shown synergism with gemcitabine in pancreatic cell lines, supporting the concept of combination therapy using everolimus in combination with intravesical gemcitabine [20]. Everolimus also enhances gemcitabine cytotoxicity in bladder cancer cell lines [21].

Thus, the objective of the study was to determine the efficacy of combination therapy with intravesical gemcitabine and oral everolimus in patients with BCG-refractory or BCG-intolerant urothelial cancer and for whom a cystectomy was recommended as standard management, but was refused.

\section{MATERIAL AND METHODS}

\section{Patients}

Patients over the age of 18 who had pathologically confirmed BCG-refractory Tis alone or Tis plus Ta or $\mathrm{T} 1$, and had adequate bone marrow, renal, and liver function were eligible to participate in the trial. BCG-refractory disease was defined as follows: persistent or recurrence of cis 6 months after one or two cycles of BCG or recurrence of cis while on maintenance. The protocol was subsequently amended to include patients with BCG-relapse bladder cancer, irrespective of the date of the original induction course of BCG and patients with BCG-intolerant disease. Patients who were receiving anticancer therapies or had major surgery within 4 wk of starting the trial, were diagnosed with other untreated malignancies, or had impaired lung or gastrointestinal function were not eligible. Patients were recruited from the outpatient clinics of the Urology and Genitourinary Oncology Services at Memorial Sloan Kettering Cancer Center (MSKCC) from December 2010 to March 2015. Investigators obtained informed consent from each patient on study.

\section{Trial design and objectives}

This was an IRB approved phase I/II trial conducted at MSKCC. In the phase I part of the trial, patients were enrolled to determine the maximum tolerated dose of everolimus. A standard $3+3$ design was utilized. The trial was designed with three dose levels such that if excessive toxicity was not evident at the highest level-ie, standard systemic dose of $10 \mathrm{mg}$ daily-further escalation would not be pursued. Phase II was designed to enroll 33 patients. If there were seven or more who were disease free after $1 \mathrm{yr}$, the treatment would be declared effective and worthy of further testing.

In phase I of the trial, patients received one of three dose levels of continuous daily oral everolimus at three dose levels: level $1-5 \mathrm{mg}$ every other $\mathrm{d}$, level 
2-5 mg daily, and level 3-10 mg daily) in combination with a fixed dose of intravesical gemcitabine at a dose of $2000 \mathrm{mg}$ twice weekly for 3 weeks, followed by a week of rest from gemcitabine. This schedule was repeated for another 28-day cycle. In brief, three patients were enrolled at the lowest dose level. If none of the three subjects experienced a dose limiting toxicity (DLT), dosage was escalated to the next highest dose level and up to three additional patients were enrolled. If one of three subjects experienced a DLT, dosage was unchanged and up to three additional subjects were enrolled. If one of six subjects experienced a DLT, the dosage was escalated to the next highest dose level and up to three more patients were enrolled. If two or three subjects in the first three subjects or $\leq 2$ out of six subjects experienced a DLT, the dose was de-escalated to a lower dose level and up to six more subjects were enrolled. DLTs were defined as follows using the NCI Common Terminology Criteria for Adverse Events (CTCAE) version 4.0: any evidence of grade 3 or 4 toxicity under Renal and Urinary Disorders in CTCAE version 4.0, Febrile neutropenia (absolute neutrophil count (ANC) $<1000 / \mu \mathrm{L}$ plus oral temperature to $38.5^{\circ} \mathrm{C}$ ), grade 4 neutropenia (ANC $<1000 / \mu \mathrm{L}$ ) for $\geq 7 \mathrm{~d}$, other grade 4 toxicity, other nonhematologic grade 3 or 4 treatment-related toxicity, excluding nausea/vomiting, rash, untreated hyperlipidemia or grade 3 fatigue lasting $\geq 7 \mathrm{~d}$, and any nonhematologic toxicity that does not resolve to grade 1 and requires treatment delay for $>7 \mathrm{~d}$ during cycle 1 . A total of six patients who received the $10 \mathrm{mg}$ dose of everolimus were eligible to be carried over to phase II of the trial.

In phase II of the trial, patients received continuous everolimus administered at the dose determined from phase I of the study along with intravesical gemcitabine at a dose of $2000 \mathrm{mg}$ twice weekly for 3 weeks followed by a week of rest from gemcitabine. This schedule was repeated for another 28-day cycle. Patients underwent a re-staging cystoscopy with narrow-band imaging and cytology every 3 mo. Random biopsies were not performed if cystoscopy with narrow band imaging and cytology were negative. Everolimus was continued for 12 months in patients who achieved a complete response (CR). Patients who demonstrated a CR (ie, negative cystoscopy and negative cytology) at their 12 month cystoscopy were observed with serial cystoscopies every 3 mo.

Patients on trial were continuously monitored for excessive toxicities to confirm the safety of the regimen and pill diaries were maintained and pill bottles were brought back to ensure compliance.
Unacceptable toxicity was defined as any grade 3 or higher local toxicity including hematuria, urgency, and frequency. Early stopping rules were defined as excessive toxicity in one of the first seven patients, two in the first 16 patients, three in the first 28 patients, and four in all 33 patients. The primary endpoint was the proportion of patients who were disease free at one year following the start of therapy. A phase II study completed at our institution evaluated patients with superficial bladder cancer who were refractory or intolerant to intravesical BCG and treated with gemcitabine intravesically. Patients in this study were enrolled from 2000 to 2004, yielding 30 evaluable patients. Based on the data from this trial, $10 \%$ of the patients treated were disease free $1 \mathrm{yr}$ following therapy. Therefore, a rate of $30 \%$ was considered promising for this trial. We have chosen a single-stage design and set the type 1 and type II error rates at 0.05 and 0.10 , respectively. The treatment would be considered effective if 7 or more patients out of the targeted enrollment of 33 were disease-free at one year. Secondary endpoints were to estimate the proportion of CR at any time within the $\mathrm{yr}$ following the start of therapy, with a binomial exact CI. Kaplan-Meier methods were planned to assess overall survival and recurrence-free progression; however, only one death was observed and a formal analysis of survival was omitted.

\section{RESULTS}

A total of 14 patients were enrolled in phase I part of the trial. Eight patients were enrolled at the first dose level of everolimus at $5 \mathrm{mg}$ every other day. One patient experienced a DLT (grade 3 diarrhea) and another patient experienced a dose delay of more than 21 days due to abdominal pain. Since those two patients were removed early from study, two replacement patients were enrolled at this dose level. Three patients were enrolled to the second dose level of everolimus and experienced no DLTs. The final three patients enrolled at the highest dose level experienced no DLTs and thus were also evaluable for the phase II efficacy assessment part of the study. The recommended phase II dose was determined to be $10 \mathrm{mg}$ daily of everolimus. Patient characteristics including protocol accrual numbers are outlined in Table 1.

A total of 23 patients were enrolled in the phase II part of the trial and 19 were evaluable for primary and secondary endpoints. There were 8 patients who withdrew consent from the study, four of which 
Table 1

Patient characteristics of phase I/II cohort

\begin{tabular}{lc}
\hline Phase I & \\
Total enrollment (N) & 14 \\
Age, yr, median (IQR) & $68(64-72)$ \\
Male & $12(86 \%)$ \\
Phase II & \\
Total enrollment (N) & 23 \\
Evaluable patients for response & 19 \\
Age, yr, median (IQR) & $66(61-74)$ \\
Male & $20(87 \%)$ \\
Rounds of Prior BCG, median (IQR) & $2(1,2)$ \\
1 & $6(32 \%)$ \\
2 & $9(47 \%)$ \\
3 & $2(11 \%)$ \\
4 & $2(11 \%)$ \\
Type of BCG failure & \\
BCG-Refractory & $11(58 \%)$ \\
BCG-Recurrence & $5(26 \%)$ \\
BCG-Intolerant & $2(11 \%)$ \\
CIS on Maintenance & $1(5.3 \%)$ \\
Cystectomy (N) & 9 \\
pTIS & $5(50 \%)$ \\
$<$ pT2 & $2(25 \%)$ \\
>pT2 & $2(25 \%)$ \\
Total inevaluable patients & $4(17 \%)$ \\
\hline
\end{tabular}

$\mathrm{IQR}=$ interquartile range.

did not begin treatment and were not evaluated for response. Four patients withdrew consent after beginning treatment and were considered as treatment failures due to early withdrawal. Of those who began treatment 6 patients were removed from trial early due to excessive toxicity $(n=3)$, other complicating disease $(n=1)$, protocol violation $(n=1)$, and a dose delay of $>21$ days $(n=1)$ and were also considered as treatment failures. Although there was an insufficient number of patients that met the specific toxicity criteria as defined by the early stopping rule, the trial was terminated early $(\mathrm{N}=23)$ at the discretion of the principal investigator based on the excessive number of patients requesting withdrawal from the study during the maintenance phase of treatment due to non-organ specific toxicities. Patient accrual and demographic details for the phase II part of the trial is listed in Table 1. All toxicities experienced during the phase II of the trial that were either probably or definitely related to study treatment are represented in Table 2 , stratified by category of toxicity. The majority of the grade 3 and 4 toxicities were metabolic. Everolimus was stopped and medical treatment was instituted. Everolimus was restarted if the laboratory values normalized within 3 weeks. Out of the 19 evaluable patients 16 failed treatment. The probability of RFS was $58 \%$ (95\% CI $33 \%-76 \%$ ) at 3 months, $27 \%$ (95\% CI 9\%-49\%) at both 6 and 9 months, and
Table 2

Toxicities experienced during the trial that were definitely or probably related to study treatment. Patients were included in this table once for each type of toxicity they experienced under the highest grade reported for that toxicity. All toxicities are categorized by System Organ Class as defined by the Common Terminology Criteria for Adverse Events (CTCAE) Version 4.0

\begin{tabular}{lcccc}
\hline System Organ Class & Grade 1 & Grade 2 & Grade 3 & Grade 4 \\
\hline Blood Disorders & 8 & 4 & 0 & 0 \\
Endocrine Disorders & 3 & 0 & 0 & 0 \\
Gastrointestinal Disorders & 21 & 4 & 0 & 0 \\
General Disorders & 8 & 3 & 0 & 0 \\
Infections & 2 & 0 & 0 & 0 \\
Injury or Procedural & 0 & 2 & 0 & 0 \\
$\quad$ Complication & & & & \\
Investigations & 40 & 6 & 4 & 0 \\
Metabolism Disorders & 24 & 10 & 5 & 1 \\
Musculoskeletal Disorders & 1 & 0 & 0 & 0 \\
Nervous System Disorders & 8 & 1 & 0 & 0 \\
Reproductive System & 1 & 0 & 0 & 0 \\
$\quad$ Disorders & & & & \\
Respiratory Disorders & 6 & 1 & 1 & 0 \\
Skin Disorders & 25 & 3 & 0 & 0 \\
$\quad$ Urinary Disorders & 16 & 8 & 0 & 0 \\
Vascular Disorders & 0 & 0 & 1 & 0 \\
\hline$\quad$
\end{tabular}

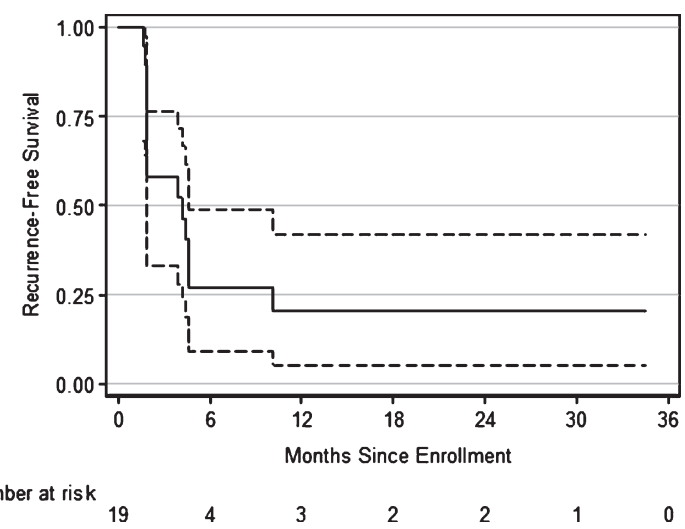

Fig. 1. Kaplan-Meier estimates of recurrence-free survival following treatment with everolimus and gemcitabine for bacillus Calmette-Guérin-refractory bladder cancer. At 1 yr, 16\% (95\% CI $5 \%-42 \%$ ) were disease free.

$20 \%$ (95\% CI 5\%-42\%) at 12 months respectively. The Kaplan-Meier estimates of RFS are shown in Fig. 1.

\section{DISCUSSION}

We have previously demonstrated the efficacy of intravesical gemcitabine in patients whose disease did not adequately respond to intravesical BCG. Initial activity was encouraging; $50 \%$ of patients achieved a CR and 23\% demonstrated a PR. However, the majority of patients relapsed within $12 \mathrm{mo}$. The 
1-yr RFS for patients with a CR was $21 \%$ (95\% CI $0 \%-43 \%$ ) [16]. The current study was designed on the premise that multiple agents may be more successful than just using a single agent, a premise pertinent to all cancers including germ cell cancers which is the most chemotherapy sensitive. The most common treatments for metastatic urothelial cancer involve regimens consisting of gemcitabine plus cisplatin [22] or the four drug combination methotrexate, vinblastine, doxorubicin plus cisplatin [23]. The biological rationale for this trial is in vitro evidence demonstrating marked synergism when gemcitabine and everolimus are given in combination [20]. Studies combining chemotherapy and tyrosine kinase inhibitors have been compromised by toxicities when both agents are given systemically. It was the hope that intravesical gemcitabine, in which very little systemic absorption is evident, would allow both.

Among evaluable patients, this trial showed promise of being effective with a rate of RFS of $20 \%$ at $1 \mathrm{yr}$, albeit with low rates of study completion. However, since the trial was terminated prematurely due to the high withdrawal of consent for reasons associated with toxicity, the clinical utility of adding everolimus to intravesical gemcitabine in this patient setting is limited. Other combination therapies have been investigated in this patient population, including the combination of BCG with interferon [24], with encouraging results. A combination of intravesical gemcitabine and mitomycin C reported a $30 \%$ RFS at $26 \mathrm{mo}$ [25] and, in a separate study, a 37\% durable response at $22 \mathrm{mo}$ [26].

In evaluating salvage therapies for patients previously treated with $\mathrm{BCG}$, comparisons between therapies are seriously hampered by the lack of standard definitions for BCG failure and BCG-refractory urothelial carcinoma and the methods of reporting the results. Thus, the results of intravesical BCG and interferon-alpha [24] or the results of intravesical gemcitabine and mitomycin-C $[25,26]$ could not be compared to the results reported in our trial. Initially, we defined patients with BCG-refractory bladder cancer as having 1) persistent disease after two consecutive courses of BCG, 2) recurrent cis less than 6 mo of achieving a response, or 3 ) recurrent cis while on maintenance. There has been a recent consensus definition of BCG unresponsive disease [27] which has been further clarified by Lerner et al. [28]. Due to limited accrual, the protocol was subsequently amended to include patients with BCG-relapse bladder cancer, irrespective of the date of the original induction course of BCG and patients with BCG-intolerant disease. Furthermore, all patients had residual cis which was used as a marker lesion to evaluate response. In contrast, the previously mentioned studies either included patients with BCG-naïve bladder cancer [24, 25] or patients with completely resected disease [26].

Fourteen patients underwent a radical cystectomy for persistent cis or for progression of disease. Three patients progressed to $\mathrm{T} 1$, one to $\mathrm{pT} 2$ and one to pT3. Of the patients who underwent radical cystectomy, five participated in phase I whereas the remaining nine participated in phase II of the trial. Daher et al. reported a 5-yr cumulative incidence of progression to $\geq \mathrm{cT} 1$ of $45 \%$ and to $\geq \mathrm{cT} 2$ in $17 \%$ for patients with newly diagnosed primary cis [29]. Herr et al. reported $82 \%$ progression among patients with BCG-refractory bladder cancer and 33\% progression among patients with BCG-relapsing bladder cancer in a cohort who were treated with mycobacterial cell wall extract [30]. Radical cystectomy remains the recommended treatment for patients who have disease that fails to respond to intravesical BCG, particularly in patients who have BCG- refractory disease as defined above. Investigational therapies should be restricted to patients who are not candidates for a major surgical intervention, and for those who refuse cystectomy.

This study was closed prematurely which is a major limitation of this study due to poor tolerance of therapy evidenced by a high number of patients requesting withdrawal from the trial. Despite this high withdrawal number, 53\% of the patients evaluable for toxicity developed Grade 3/4 toxicity similar to other reported series in metastatic disease patients with a treatment discontinuation rate of $37 \%$. Milowsky et al. observed grade 3/4 toxicities in 64\% patients and treatment was discontinued in only $17 \%$ of patients [17]. Motzer et al. reported $10 \%$ treatment discontinuation in patients receiving everolimus for metastatic renal cell carcinoma [18]. The high proportion rate of patients who stopped treatment led us to conclude that patients with potentially curable nonmuscle invasive bladder cancer (NMIBC) may be far less willing to tolerate toxicity at the level considered reasonable than patients with metastatic disease who have very poor rates of survival. This observation will be important as new tyrosine kinase inhibitors, active and considered tolerable in the metastatic disease patient setting, experience toxicity considered to be potentially excessive in the NMIBC setting. There are several limitations to this study which include premature closing, long accrual time, inability to perform 
testing for phospho-S6, phospho-4E-BP1 or sequencing due to inadequate amount of tissue.

In summary, because of the premature termination of the trial we failed to demonstrate a benefit in adding everolimus to intravesical gemcitabine. The combination of systemic oral everolimus with intravesical gemcitabine does not enhance the benefit seen with intravesical gemcitabine alone. Everolimus is associated with significant toxicity which is not well tolerated in patients with NMIBC. Trials in this patient population should be conducted with great care due to the high rate of progression of disease and patients should be made aware that cystectomy is still the standard of care.

\section{ACKNOWLEDGMENTS}

This study was supported by the following: The Sidney Kimmel Center for Prostate and Urologic Cancers, Novartis, and the NIH/NCI Cancer Center Support Grant P30 CA008748.

\section{CONFLICT OF INTEREST}

The authors have no conflict to report.

\section{REFERENCES}

[1] Siegel RL, Miller KD, Jemal A. Cancer statistics, 2016. CA Cancer J Clin 2016;66:7-30.

[2] Reuter VE, Melamed MR. The lower urinary tract. Diagn Surg Pathol 1994:1767-805.

[3] Soloway MS. Overview of treatment of superficial bladder cancer. Urology 1985;26:18-26.

[4] Dalbagni G, Herr HW. Current use and questions concerning intravesical bladder cancer group for superficial bladder cancer. Urol Clin North Am 2000;27:137-46.

[5] Lamm DL, Blumenstein BA, Crawford ED, Montie JE, Scardino P, Grossman HB, et al. A randomized trial of intravesical doxorubicin and immunotherapy with bacille Calmette-Guérin for transitional-cell carcinoma of the bladder. N Eng J Med 1991;325:1205-9.

[6] Rintala E, Jauhiainen K, Alsthan O, Hansson E, Juusela $\mathrm{H}$, Kanerva Korhonen $\mathrm{KH}$, et al. Intravesical chemotherapy (mitomycin C) versus immunotherapy (bacillus CalmetteGuerin) in superficial bladder cancer. Eur Urol 1991;20: 19-25.

[7] Vegt PDJ, Witjes JA, Witjes WPJ, Doesburg WH, Debruyne FMJ, van der Meijden APM, et al. Original articles: Bladder cancer: A randomized study of intravesical mitomycin $\mathrm{C}$, bacillus calmette-guerin tice and bacillus calmette-guerin RIVM treatment in pTa-pT1 papillary carcinoma and carcinoma in situ of the bladder. J Urol 1995;153:929-33.

[8] Krege S, Giani G, Meyer R, Otto T, Rübben H, Noll F, et al. A randomized multicenter trial of adjuvant therapy in superficial bladder cancer: Transurethral resection only versus transurethral resection plus mitomycin $\mathrm{C}$ versus transurethral resection plus bacillus Calmette- Guerin. J Urol 1996;156:962-6.

[9] Lundholm C, Norlén BJ, Ekman P, Jahnson S, Lagerkvist $\mathrm{M}, \mathrm{Lindeborg} \mathrm{T}$, et al. A randomized prospective study comparing long-term intravesical instillations of mitomycin $\mathrm{C}$ and bacillus Calmette-Guerin in patients with superficial bladder carcinoma. J Urol 1996;156:372-6.

[10] Malmström PU, Wijkström H, Lundholm C, Wester K, Busch C, Norlén BJ, et al. 5-Year followup of a randomized prospective study comparing mitomycin $\mathrm{C}$ and bacillus Calmette-Guerin in patients with superficial bladder carcinoma. J Urol 1999;161:1124-7.

[11] Hudson MA, Herr HW. Carcinoma in situ of the bladder. J Urol 1995;153:564-72.

[12] Herr HW, Badalament RA, Amato DA, Laudone VP, Fair WR, Whitmore Jr WF, et al. Superficial bladder cancer treated with bacillus Calmette-Guerin: A multivariate analysis of factors affecting tumor progression. J Urol 1989;141:22-9.

[13] Burris HA 3rd, Moore MJ, Andersen J, Green MR, Rothenberg ML, Modiano MR, et al. Improvements in survival and clinical benefit with gemcitabine as first-line therapy for patients with advanced pancreas cancer: A randomized trial. J Clin Oncol 1997;15:2403-13.

[14] Stadler WM, Kuzel T, Roth B, Raghavan D, Dorr FA. Phase II study of single-agent gemcitabine in previously untreated patients with metastatic urothelial cancer. J Clin Oncol 1997;15:3394-8.

[15] Lorusso V, Pollera CF, Antimi M, Luporini G, Gridelli C, Frassineti GL, et al. A phase II study of gemcitabine in patients with transitional cell carcinoma of the urinary tract previously treated with platinum. Italian Co-operative Group on Bladder Cancer. Eur J Cancer 1998;34:1208-12.

[16] Dalbagni G, Russo P, Bochner B, Ben-Porat L, Sheinfeld $\mathrm{J}$, Sogani $\mathrm{P}$, et al. Phase II trial of intravesical gemcitabine in bacille Calmette-Guerin-refractory transitional cell carcinoma of the bladder. J Clin Oncol 2006;24:2729-34.

[17] Milowsky MI, Iyer G, Regazzi AM, Al-Ahmadie H, Gerst SR, Ostrovnaya I, et al. Phase II study of everolimus in metastatic urothelial cancer. BJU Int 2013;112:462-70.

[18] Motzer RJ, Escudier B, Oudard S, Hutson TE, Porta C, Bracarda S, et al. Efficacy of everolimus in advanced renal cell carcinoma: A double-blind, randomised, placebo-controlled phase III trial. Lancet 2008;372:449-56.

[19] Chiong E, Lee IL, Dadbin A, Sabichi AL, Harris L, Urbauer D, et al. Effects of mTOR inhibitor everolimus (RAD001) on bladder cancer cells. Clin Cancer Res 2011;17:2863-73.

[20] Tuncyurek P, Mayer JM, Klug F, Dillmann S, Henne-Bruns D, Keller F, et al. Everolimus and mycophenolate mofetil sensitize human pancreatic cancer cells to gemcitabine in vitro: A novel adjunct to standard chemotherapy? Eur Surg Res 2007;39:380-7.

[21] Pinto-Leite R, Arantes-Rodrigues R, Palmeira C, Gaivao I, Cardoso ML, Colaco A, et al. Everolimus enhances gemcitabine-induced cytotoxicity in bladder-cancer cell lines. J Toxicol Environ Health A 2012;75:788-99.

[22] von der Maase H, Sengelov L, Roberts JT, Ricci S, Dogliotti $\mathrm{L}$, Oliver T, et al. Long-term survival results of a randomized trial comparing gemcitabine plus cisplatin, with methotrexate, vinblastine, doxorubicin, plus cisplatin in patients with bladder cancer. J Clin Oncol 2005;23:4602-8.

[23] Sternberg CN, de Mulder P, Schornagel JH, Theodore C, Fossa SD, van Oosterom AT, et al. Seven year update of an EORTC phase III trial of high-dose intensity 
M-VAC chemotherapy and G-CSF versus classic M-VAC in advanced urothelial tract tumours. Eur J Cancer 2006; 42:50-4.

[24] O’Donnell MA, Krohn J, DeWolf WC. Salvage intravesical therapy with interferon-alpha $2 \mathrm{~b}$ plus low dose bacillus Calmette-Guerin is effective in patients with superficial bladder cancer in whom bacillus Calmette-Guerin alone previously failed. J Urol 2001;166:1300-4, discussion 4-5.

[25] Lightfoot AJ, Breyer BN, Rosevear HM, Erickson BA, Konety BR, O'Donnell MA. Multi-institutional analysis of sequential intravesical gemcitabine and mitomycin $\mathrm{C}$ chemotherapy for non-muscle invasive bladder cancer. Urol Oncol 2014;32:35.e15-9.

[26] Cockerill PA, Knoedler JJ, Frank I, Tarrell R, Karnes RJ. Intravesical gemcitabine in combination with mitomycin $\mathrm{C}$ as salvage treatment in recurrent non-muscle-invasive bladder cancer. BJU Int 2016;117:456-62.
[27] Jarow JP, Lerner SP, Kluetz PG, Liu K, Sridhara R, Bajorin $\mathrm{D}$, et al. Clinical trial design for the development of new therapies for nonmuscle-invasive bladder cancer: Report of a Food and Drug Administration and American Urological Association public workshop. Urology 2014;83:262-4.

[28] Lerner SP, Dinney C, Kamat A, Bivalacqua TJ, Nielsen M, O'Donnell M, et al. Clarification of bladder cancer disease states following treatment of patients with intravesical BCG. Bladder Cancer 2015;1:29-30.

[29] Chade DC, Shariat SF, Godoy G, Savage CJ, Cronin AM, Bochner $\mathrm{BH}$, et al. Clinical outcomes of primary bladder carcinoma in situ in a contemporary series. J Urol 2010;184:74-80.

[30] Herr HW, Milan TN, Dalbagni G. BCG-refractory vs. BCG-relapsing non-muscle-invasive bladder cancer: A prospective cohort outcomes study. Urol Oncol 2015;33:108.e1-4. 